\title{
Representaciones, lenguaje, conversión, símbolos, semiótica, narrativas simbólicas... ¿què tienen que ver con la comprensión en matemáticas? ${ }^{1}$
}

Representations, language conversion, symbols, semiotics, symbolic narratives ... What have they to do with understanding in mathematics?

Representações, a conversão da linguagem, símbolos, semiótica, narrativas simbólicas ... O que eles têm a ver com a compreensão em matemática?

Recibido: mayo de 2013

Gloria Inés Neira Sanabria ${ }^{2}$

Aceptado: agosto de 2013

\begin{abstract}
Resumen
¿Es la matemática un lenguaje?, ¿El simbolismo matemático es necesario e imprescindible para aprender matemáticas?, Cuando se modela un enunciado del lenguaje verbal en símbolos, ¿se puede decir que se está "traduciendo" de un lenguaje a otro? ¿Aprender matemáticas es ser capaz de entender el lenguaje matemático y saberlo traducir, ó es más que eso?, ¿el problema de simbolizar o modelar es una traducción? ¿Qué relación es posible plantear entre el estudio de la traducción al lenguaje simbólico y los obstáculos didácticos en la enseñanza de un área especifica de la matemática, por ejemplo, el álgebra o el cálculo? Parafraseando a Kline, "El símbolo expresa una idea, oculta una idea, u oculta la ausencia de una idea?"
\end{abstract}

Palabras clave: Otras nociones de Educación matemática; sistemas de representación; semiótica; comprensión en matemáticas.

\begin{abstract}
Mathematics Is language?, Is mathematical symbolism is necessary and essential for learning mathematics?, When modeling a statement of verbal symbols, can say that it is "translating" from one language to another? Will learning mathematics is to be able to understand mathematical language and translate know, or is it more than that?, Does symbolize or modeling problem is a translation? What relationship can pose between the study of symbolic language translation and educational obstacles in teaching a specific area of mathematics, for example, algebra or calculus? Paraphrasing Kline, "The symbol expresses an idea, an idea hidden, or hides the absence of an idea?".
\end{abstract}

Keywords: Other notions of mathematics education, systems of representation, semiotics mathematical understanding.

\footnotetext{
1 Artículo de Investigación

2 Universidad Distrital Francisco Jose de Caldas. Bogotá, Colombia. Contacto:gneira@udistrital.edu.co
} 


\section{Resumo}

Matemática é linguagem?, É o simbolismo matemático é necessário e essencial para a aprendizagem da matemática?, Ao modelar uma declaração de símbolos verbais, pode-se dizer que ele está "traduzindo" a partir de uma língua para outra? Will aprendizagem da matemática é ser capaz de entender a linguagem matemática e traduzir sabe, ou é mais do que isso?, O problema simbolizam ou modelagem é uma tradução? Que relação pode representar entre o estudo da tradução da linguagem simbólica e obstáculos educacionais no ensino de uma área específica de matemática, por exemplo, álgebra ou cálculo? Parafraseando Kline, "O símbolo exprime uma idéia, uma idéia oculta, ou esconde a ausência de uma idéia?"

Palavras-chave: Outras noções de educação matemática, sistemas de representação semiótica compreensão matemática.

\section{Presentacion del problema}

En la perspectiva de Duval y compartida por la autora de este escrito, las matemáticas se distinguen de otras áreas del conocimiento, entre otros aspectos, en que el único acceso a ellas es de naturaleza semiótica, vía la representación; Cada representación no presenta las mismas propiedades o características del objeto y recíprocamente, ninguna representación es completa y adecuada al objeto. Además, según la naturaleza del sistema productor de la representación, y el modo fenomenológico de producción de éstas, se habla entonces de representaciones semióticas, cuya producción es intencional, para distinguirlas de las representaciones neurales, cuya producción no es intencional sino automática, en tanto se involucran procesos motores y neuronales.

Desde esta perspectiva se postula que las representaciones en matemáticas son de naturaleza semiótica y que toda actividad matemática implica el recurso a representaciones semióticas porque los objetos estudiados no son accesibles perceptiva o instrumentalmente, como en otros ámbitos de conocimiento científico, es decir, sólo se puede acceder a los objetos matemáticos mediante sus representaciones, y no se puede confundir el objeto con su representación.

\section{Marco de referencia}

Según Duval, las transformaciones de representaciones se pueden clasificar en dos tipos. Por una parte, el Tratamiento, como transformación de una representación en otra representación de un mismo registro, y por otro lado, la Conversión, como transformación de la representación de un objeto en un registro en otra representación del mismo objeto en otro registro. En cuanto al aprendizaje, la teoría de Duval es radical al postular la comprensión en matemáticas como la habilidad para articular registros de representación, es decir se postula la conversión como el paso crucial en la comprensión. Cuando se le brinda al estudiante una pluralidad de representaciones del mismo objeto matemático, se están brindando mayores posibilidades de comprensión de ese objeto, pero no basta la multi-representación, es decir no es suficiente con representar de diversas maneras un objeto, si no se garantiza mediante el trabajo cognitivo, el reconocimiento del mismo objeto en los diferentes registros. Por ejemplo el objeto "mitad", se puede representar como $1 / 2$, o como el $50 \%$, o como "la mitad de" o como un cuadrado divido en dos partes y sombreada una de ellas.

Acerca de la comprensión de los estudiantes: Duval manifiesta la importancia que tiene para los profesores el hecho de que los alumnos usen diferentes 
representaciones en el aprendizaje de conceptos matemáticos pero a la vez resalta que el tema principal es saber cuáles son los tipos de tareas y actividades para lograr este propósito.

Al parecer la idea más obvia es exponer varias posibles representaciones al mismo tiempo. Pero, desde un punto de vista didáctico estas actividades no conducen a ninguna parte, dado que toda representación comporta dos dimensiones semánticas: la del contenido que representa, y que es intrínseca al registro movilizado, y la del objeto que representa, que es independiente del registro que se moviliza.

Por ejemplo el contenido de una representación gráfica puede ser una recta, una parábola, un círculo, etc., que son tres contenidos visualmente diferentes y representan tres objetos matemáticos: una función lineal, una cuadrática y una relación que no es una función pero que caracteriza un objeto geométrico, como es el círculo. Se dice además que la yuxtaposición de dos representaciones de un mismo objeto en dos registros diferentes no puede resolver el problema cognitivo del reconocimiento del mismo objeto representado, porque las diferencias de contenido de las representaciones varían independientemente de los objetos representados. Así aparecen dos situaciones de reconocimiento en cierta manera opuestas: reconocer el mismo objeto en dos representaciones cuyos contenidos son muy diferentes porque corresponden a dos registros diferentes, por ejemplo una ecuación de primer grado y el grafo de una recta, y reconocer dos objetos diferentes en dos representaciones cuyos contenidos parecen semejantes porque corresponden al mismo registro, como por ejemplo dos grafos que son visualmente rectas o parábolas, o entre dos enunciados de problemas que utilizan las mismas palabras y describen la misma situación real (como por ejemplo los problemas aditivos o los problemas de proporcionalidad, etc.).

\section{Metodología}

En la temática que nos ocupa del lenguaje y las representaciones, y con el propósito de presentar otra mirada no porque la anterior presente debilidades, otros investigadores como Radford, plantean el tránsito de la nominación "traducir", usada tantas veces para significar el paso del lenguaje natural al lenguaje simbólico por el término "narrativa simbólica". Una búsqueda rápida de lo que subyace en el término narrativa nos arroja lo siguiente: las estructuras narrativas son estructuras lineales, donde hay a grandes rasgos una sola voz, o donde las voces corean al unísono, o al menos, en la misma dirección. La estructura narrativa central es el relato, pero se considera también, como estructura del orden narrativo, a la descripción. No implica solamente pasar al lenguaje de símbolos, sino que la palabra narrativa implica una nueva manera de contar, de relatar, de describir, de interpretar, con unas reglas propias y diferentes de la anterior forma de narrar: se pasa, en efecto a un espacio semiótico nuevo.

Por ejemplo, abrir el paso a esa nueva narrativa implica darle giros a una frase del estilo "Kelly tiene dos dulces más que Manuel" pero darle tantas vueltas y re-decirla hasta llegar a una frase asertiva del tipo: "Kelly tiene lo que tenga Manuel más 2" es de una complejidad mayor que posibilita de alguna manera el paso a la solución del problema.

Los sujetos, los adjetivos cambian de una narrativa a otra, luego se comprende esta manera de nombrar lo que casi siempre hemos llamado "lenguaje de simbolos", significando con esto una traducción semántica y sintáctica directa de un lenguaje a otro, que una vez más Radford llama la atención acerca de lo erróneo de este imaginario. Es decir, nos lleva a la toma de conciencia que al cambiar de narrativa los personajes cambian, los héroes ya no son más los que eran (Camila, Manuel, José), sino que se emergen como protagonistas, como héroes las relaciones numéricas: son ellas las que pasan a ser los sujetos, porque la nueva narrativa abre un nuevo espacio semiótico.

\section{Análisis}

La actividad matemática se basó en el siguiente problema verbal corto: "Kelly tiene 2 caramelos más que Manuel. José tiene 5 caramelos más que Manuel. Todos juntos tienen 37 caramelos" [1]. El 
mismo problema verbal fue utilizado para generar tres problemas involucrando transformaciones en la expresión algebraica de los datos. En el problema 1, a los estudiantes se les pidió designar el número de caramelos de Manuel por x, elaborar una expresión simbólica para los de Kelly y José, y, luego, escribir y resolver una ecuación correspondiente al problema verbal. Los problemas 2 y 3 incluyen cuestiones similares. La diferencia es que, en el Problema 2, a los estudiantes se les pidió que designaran el número de caramelos de Kelly por $\mathrm{x}$, mientras que en el Problema 3, se les pidió que designaran del número de caramelos de José por $\mathrm{x}$.

El análisis de un problema tan sencillo como el de la experiencia que relata, tan común en todos los países, los textos y las aulas, deja ver que con toda razón hay dificultades y obstáculos en el aprendizaje de las matemáticas, empezando por la comunicación, por la expresión de las situaciones, por la argumentación, por las "malas" inferencias". También deja entrever el origen semiótico de tales dificultades o conflictos, utilizando el término de Juan Díaz Godino, en el Enfoque Ontosemiótico de la Cognición en matemáticas, enfoque al que se hace alusión aquí solamente para enfatizar el origen semiótico de las dificultades aludidas.

. Esto nos lleva a reflexionar sobre cómo en la mayoría de casos en los que se pide a los alumnos que resuelvan problemas tipo historieta como el que se analiza en el texto, no se piensa en las implicaciones cognitivas que requiere su solución, sino que se espera que el estudiante pase casi que directamente del enunciado a la ecuación que lo resuelve.

\section{Conclusiones}

Considero que es de un valor pedagógico y didáctico muy grande las propuestas y los análisis de estos investigadores para hacer esa transición, mostrando los procesos y dificultades que se dan antes de llegar a la ecuación, como son el modo de designación de los objetos del discurso a través del simbolismo algebraico y las operaciones que se realizan sobre los símbolos que designan los objetos, aspectos a los cuales se les pone muy poca atención en la enseñanza del álgebra.

Desde el punto de vista didáctico valoro el reconocimiento de frases comparativas en el enunciado de una situación y la estrategia de transformarlas en frases asertivas para facilitar y comprender la introducción de letras para designar las cantidades desconocidas y la elaboración de expresiones simbólicas. Este análisis es muy importante para ser discutido con los docentes, pues les podría dar herramientas para ayudar a los alumnos a comprender la resolución de este tipo de problemas. Suele ocurrir, en la mayoría de los casos que el alumno debe elaborar sus propias expresiones simbólicas y operar con ellas para formar la ecuación sin mayor comprensión de los procesos involucrados, lo cual los lleva a adivinar e inventar procedimientos, muchas veces equivocados.

Los problemas de comprensión con los que tropiezan la mayoría de los estudiantes, también son muy específicos del aprendizaje de las matemáticas, porque la transferencia de conocimientos y la comprensión siempre implican la conversión de representaciones, de hecho el isomorfismo matemático entre dos representaciones nunca involucra su isomorfismo cognitivo y por tanto no puede ser reconocido por los estudiantes.

Por supuesto que la comprensión no significa dar un salto desde el contenido de la representación hasta el concepto puramente matemático representado; consiste más bien en relacionar diversos contenidos de representación del mismo concepto. La comprensión en matemáticas requiere una coordinación interna entre los diversos sistemas de representación semiótica usados y sin desarrollar tal coordinación es imposible cruzar el umbral de la conversión de representación.

De esta manera, la habilidad para movilizar diversas representaciones conjuntamente, depende del desarrollo de esta coordinación, y la comprensión conceptual no es la condición de tal coordinación, sino que surge de su desarrollo. Así, lo más importante para la enseñanza de las matemáticas no es la elección del mejor sistema de representación, sino lograr que los estudiantes sean capaces 
de relacionar muchas maneras de representar los contenidos matemáticos.

Claro que una multi-representación es deseable en nuestras aulas, en las que generalmente se trabaja con un modelo axiomático tradicional que todavía manteiene el esquema :deficion, ejemplos, ejerecicios. Cada objeto casi tiene una sola representación, en la gran generalidad de nuestras aulas de clase, así que ya de por sí postular la importancia de las diversas represesntaciones para un mismo objeto es ya importante, en cuanto cada una nos permite capturar unas u otras propiedades del objeto, pero Duval trasciende lejos esta mera enunciación al afirmar que la enseñanza debe basrse y propiciar la articulación de los registros, trabajar la conversión, favorecer el tránsito entre unas y otras y reconocer el mismo objeto en todas ellas.

\section{Referencias}

Duval, R. (2006a). Un tema crucial en la educación matemática: la habilidad para cambiar de registro de representación. La Gaceta de la RSME., 9(1), 143-168.

Duval, R. (2006b). La conversión des représentations: Un des deux processus fondamentaux de la pensée. Grenoble: Presses universitaires de Grenoble.

Radford, L. (2003). Gestures, speech, and the sprouting of signs. Mathematical Thinking and Learning, 5(1), 37-70. 